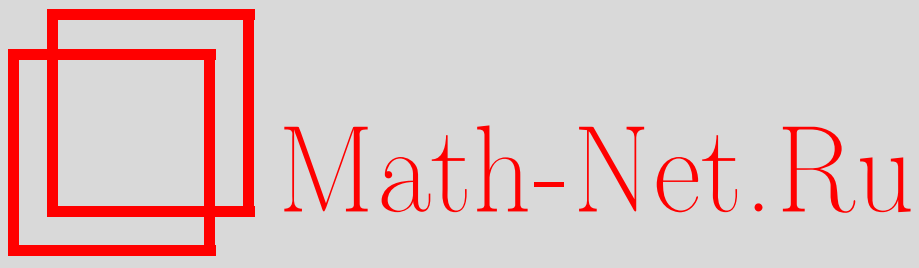

Д. П. Желобенко, Дифференциальные операторы и дифференциальное исчисление в квантовых группах, Изв. РАН. Сер. матем., 1998, том 62, выпуск 4, 25-50

DOI: https://doi.org/10.4213/im191

Использование Общероссийского математического портала Math-Net.Ru подразумевает, что вы прочитали и согласны с пользовательским соглашением

http://www . mathnet.ru/rus/agreement

Параметры загрузки:

IP : 3.89 .185 .249

26 апреля 2023 г., $18: 10: 27$ 
УДК $513.88+519.46$

Д. П. Желобенко

\title{
Дифференциальные операторы и дифференциальное исчисление в квантовых группах
}

\begin{abstract}
Исследуются абстрактные дифференциальные операторы в биалгебрах и алгебрах Хопфа (квантовых группах). Доказаны теоремы плотности и некоторые структурные теоремы для алгебр дифференциальных операторов, определяемых дуальными парами алгебр Хопфа $A, B$. Отмечена связь с квантовым дублем В. Дринфельда и с дифференциальной геометрией в квантовых группах.

Библиография: 20 наименований.
\end{abstract}

\section{Введение}

Алгебры Хопфа (квантовые группы) давно уже стали предметом интенсивного исследования, особенно с 1985 г., с тех пор, как В. Дринфельдом [8] были обнаружены новые содержательные примеры алгебр Хопфа (отличных от коммутативных или кокоммутативных). Безусловно, алгебры Хопфа представляют собой яркий пример "неоклассики" в современной математике, и не только благодаря своей замечательной родословной (включающей связь с квантовой физикой), но в значительной степени благодаря их собственному совершенству. Достаточно вспомнить дуальные пары алгебр Хопфа, ассоциированные с полупростыми комплексными алгебрами Ли (или с алгебрами Каца-Муди) [7], [9], [10], связь с уравнением Янга-Бакстера и скобками Пуассона [7], конструкцию квантового дубля В. Дринфельда [7], [9], обобщение теории двойственности Таннаки-Крейна [14], кристаллические методы Г. Люстига [13] и М. Кашивары [11], [12] в теории представлений квантовых групп, их влияние на классическую теорию представлений [17] и т.д.

В 1989 г. С. Л. Вороновичем [15] было показано, что каждая алгебра Хопфа А обладает богатой дифференциально-геометрической структурой, определяемой в терминах дифференциальных форм, ассоциированных с правыми (левыми) идеалами алгебры $A$. C тех пор появилось большое количество работ по дифференциальному исчислению в квантовых группах. В [16] была отмечена роль квантового дубля В. Дринфельда в дифференциальном исчислении, а именно в описании присоединенного действия в алгебре внешних дифференциальных форм. Для левых (правых) идеалов конечной коразмерности рассматривалась двойственность между касательными и кокасательными расслоениями в квантовых группах (см., например, [4]). По-видимому, особый интерес для дальнейших исследований представляют "квантовые алгебры Ли", ассоциированные с квантовыми группами [5].

Работа выполнена при финансовой поддержке РФФИ (грант 95-01-00233).

(C) Д.П. Желовенко 1998 
Предметом исследования в данной статье являются “дифференциальные операторы”, естественно возникающие в теории алгебр Хопфа и имеющие непосредственное отношение к дифференциальному исчислению в смысле [15]. Показано, что с каждой дуальной парой алгебр Хопфа $A, B$ естественно связаны некоторые алгебры дифференциальных операторов, имеющие тесную аналогию с классическими алгебрами Вейля (т.е. с алгебрами дифференциальных операторов в евклидовых пространствах). Показано, что дифференциальная геометрия С. Л. Вороновича естественно излагается в терминах дифференциальных операторов, т.е. левоинвариантных (правоинвариантных) векторных полей на квантовых группах. Разумеется, эта конструкция сводится к стандартной двойственности между касательными и кокасательными расслоениями в квантовых группах. С другой стороны, именно при этом подходе проясняется роль квантового дубля В. Дринфельда в дифференциальной геометрии квантовых групш.

Следует отметить, что дифференщиальные операторы в алгебрах Хопфа рассматривались эпизодически еще до появления общей концепции квантовых групп [8]. В известной мере эта конструкция относится к области математического фольклора. Одно из первых формальных определений содержится в краткой заметке С.П. Новикова [19], в связи с предложенньм им обшим понятием алгебр Милнора.

План изложения в данной статье сводится к следующему. В $\S 1$ определяются алгебры дифференциальных операторов в биалгебрах и квантовых группах. В 22 для этих алгебр устанавливаются некоторые аналоги теоремы плотности Джекобсона. В $\S 3$ исследуются структурные свойства дифференциальных алгебр. В $\S 4$ рассматривается действие квантового дубля В. Дринфельда, ассоциированного с дуальной парой алгебр Хопфа. В $\S 5$ излагается общая схема дифференциального исчисления в терминах векторных полей. В $§ 6$ устанавливается связь этой схемы с дифференциальным исчислением С. Л. Вороновича. В $\S 7$ изложены элементарные свойства дифференциальных операторов, связанные со слабыми топологиями в алгебрах Хопфа.

Таким образом, содержание этой статьи сводится к элементарному введению в дифференциальное исчисление на квантовых группах. В известной степени эта статья может рассматриваться как продолжение конструкции [3], связанной с описанием дифференщиальных операторов в градуированных алгебрах. Связь с этой конструкцией рассматривается в $\S 3$.

Краткое изложение результатов статьи можно найти в [18]. См. также [20].

\section{§ 1. Алгебра $D(A)$}

1.1. Пусть $A$ - биалгебра над полем $k$. Напомним, что $A$ есть ассоциативная алгебра с единицей, снабженная коумножением $\Delta: A \rightarrow A \otimes A$ и коединицей $\varepsilon: A \rightarrow k$, где $\Delta, \varepsilon$ суть морфизмы алгебр с единищей, связанные условием коассоциативности

$$
(\Delta \otimes 1) \Delta=(1 \otimes \Delta) \Delta
$$


и условием согласованности

$$
(\varepsilon \otimes 1) \Delta=(1 \otimes \varepsilon) \Delta=1 .
$$

Здесь тензорные степени алгебры $A$ рассматриваются как алгебры (с покомпонентным умножением) и каждая из алгебр $A \otimes 1,1 \otimes A$ отождествляется с алгеброй $A$.

Условие коассоциативности позволяет определить индуктивные "степени" оператора $\Delta$, в том числе морфизм $\Delta^{2}: A \rightarrow A \otimes A \otimes A$, определяемый равенством (1.1). В дальнейшем мы будем использовать следующие символы Шведлера:

$$
\Delta x=x_{1} \otimes x_{2}, \quad \Delta^{2} x=x_{1} \otimes x_{2} \otimes x_{3}
$$

(заменяюшие сумму слагаемых вида $x_{1}^{i} \otimes x_{2}^{i}$ и т.д.). Условие (1.2) в этих обозначениях принимает следующий вид:

$$
\varepsilon\left(x_{1}\right) x_{2}=x_{1} \varepsilon\left(x_{2}\right)=x
$$

для всех $x \in A$.

Биалгебра $A$ называется алгеброй Xопфа, если в ней определен обратимый $а н-$ типод $\gamma: A \rightarrow A$, удовлетворяюший соотношению

$$
\gamma\left(x_{1}\right) x_{2}=x_{1} \gamma\left(x_{2}\right)=\varepsilon(x)
$$

для всех $x \in A$.

Здесь $\gamma$ определяется первоначально как эндоморфизм векторного пространства $A$. Однако из условия (1.5) следует, что $\gamma$ есть антиавтоморфизм алгебры $A$ (см., например, [10]).

1.2. Биалгебры $A, B$ над полем $k$ называются дуальными друг другу, если существует невырожденная билинейная форма $\langle.,$.$\rangle на A \otimes B$, удовлетворяюшая следуюшим соотношениям двойственности:

$$
\begin{array}{ll}
\langle x, 1\rangle=\varepsilon(x), & \langle 1, y\rangle=\varepsilon(y), \\
\langle x, y z\rangle=\langle\Delta x, y \otimes z\rangle, & \langle x y, z\rangle=\langle x \otimes y, \Delta z\rangle
\end{array}
$$

для всех $x, y, z$ (из $A$ или $B$ ). Здесь мы используем одинаковые обозначения для операций $\Delta, \varepsilon$ в алгебрах $A$ и $B$.

Невырожденность формы $\langle.$, . $\rangle$ позволяет определить каноническое вложение $B \rightarrow A^{*}$ (аналогично $A \rightarrow B^{*}$ ), так что форма $\langle.,$.$\rangle совпадает с сужением на$ $A \otimes B$ канонической билинейной формы, определенной в $A \otimes A^{*}$. Мы будем, как правило, использовать обозначение $y(x)$ (аналогично $x(y)$ ) для значения линейного функционала $y \in A^{*}$ на элементе $x \in A$. 
Известно [7], что все дуальные биалгебры $B \subset A^{*}$ содержатся в наибольшей дуальной биалгебре $A^{+} \subset A^{*}$. Если $A$ и $B$ - алгебры Хопфа, то антиподы в $A$ и $B$ связаны соотношением сопряженности, т.е.

$$
\langle\gamma x, y\rangle=\langle x, \gamma y\rangle
$$

для всех $x \in A, y \in B$.

Напомним [7], что алгебра $A^{+}$состоит из всех функционалов $f \in A^{*}$, для которых $\mu^{*}(f) \in A^{*} \otimes A^{*}$, где $\mu^{*}: A^{*} \rightarrow(A \otimes A)^{*}$ - отображение, сопряженное к операции умножения $\mu: A \otimes A \rightarrow A$. При этом $\Delta=\mu^{*}$ есть операция коумножения в алгебре $A^{+}$.

1.3. Пусть $A, B$ - дуальные биалгебры, и пусть $a \in \operatorname{End} A, b \in$ End $B$ - операторы, сопряженные друг другу относительно формы $\langle$. , . $\rangle$, т.е.

$$
\langle a x, y\rangle=\langle x, b y\rangle
$$

для всех $x \in A, y \in B$. Из невырожденности формы $\langle.,$.$\rangle следует в этом случае$ однозначность операций сопряжения $a^{\prime}=b, b^{\prime}=a$.

$\mathrm{B}$ частности, пусть $a \in A$. Поскольку $A$ есть алгебра с единицей, мы можем отождествить элемент $a$ с оператором левого умножения $l_{a} x=a x$ в алгебре $A$. Аналогично, каждый элемент $b \in B$ отождествляется с оператором $l_{b} \in \operatorname{End} B$. Легко проверить, что каждый из этих операторов обладает сопряженным относительно формы $\langle.$, . $\rangle$. Например, имеем

$$
b^{\prime} x=b\left(x_{1}\right) x_{2}
$$

для каждого $b \in B$, где $x \in A, \Delta x=x_{1} \otimes x_{2}$. Заменяя в этом определении операторы $l_{a}$ операторами правого умножения $r_{a} x=x a$, условимся также использовать обозначения $\bar{a} x=x a, \bar{b} y=y b(x \in A, y \in B)$. Соответствующий аналог соотношения (1.10) имеет вид

$$
\bar{b}^{\prime} x=x_{1} b\left(x_{2}\right) .
$$

Используя правило двойственности (1.7), легко проверить, что каждый из операторов $\partial=b^{\prime}, \bar{b}^{\prime}$ удовлетворяет следующему тождеству Лейбница:

$$
\partial(x y)=\partial_{1}(x) \partial_{2}(y)
$$

для всех $x, y \in A$, где положено $\partial_{i}=b_{i}^{\prime}, \bar{b}_{i}^{\prime}(i=1,2)$ при $\Delta b=b_{1} \otimes b_{2}$.

Заметим также, что алгебра $A$ есть левый $B$-модуль относительно операторов $b * x=\bar{b}^{\prime} x$ и правый $B$-модуль относительно операторов $x * b=b^{\prime} x$. Таким образом, $A$ есть $B$-бимодуль. Аналогично, алгебра $B$ есть $A$-бимодуль с операторами $a * y=\bar{a}^{\prime} y, y * a=a^{\prime} y(a \in A, y \in B)$. 
1.4. ОПРЕДЕЛЕНИЕ. Пусть $A$ - биалгебра над полем $k$ с фиксированной дуальной биалгеброй $B$. Условимся отождествлять элементы $a \in A, b \in B$ с соответствуюшими операторами $l_{a}, l_{b}$, так что $A \subset \operatorname{End} A, B \subset \operatorname{End} B$, и пусть $D(A)=\operatorname{Diff}(A, B)$ - подалгебра в End $A$, порожденная подалгебрами $A, B^{\prime}$.

Заметим, что правило Лейбница (1.12) может быть записано как соотношение коммутации между операторами $\partial=b^{\prime} \in B^{\prime}, x=l_{x} \in A$, а именно

$$
\partial x=\partial_{1}(x) \partial_{2} .
$$

Используя (1.13), находим, что алгебра $D(A)$ натянута на элементы вида $a b^{\prime}$ $(a \in A, b \in B)$. Иначе говоря, имеем

$$
D(A)=A B^{\prime} .
$$

Варьируя в этом определении операторы $l_{a}, l_{b}$ с операторами $r_{a}, r_{b}$, получаем также следующие подалгебры в End $A$, родственные $D(A)$ :

$$
\bar{D}(A)=A \bar{B}^{\prime}, \quad D(\bar{A})=\bar{A} B^{\prime}, \quad \bar{D}(\bar{A})=\bar{A} \bar{B}^{\prime} .
$$

Здесь $\bar{A}, \bar{B}$ - подалгебры в End $A$, End $B$ соответственно, состоящие из операторов $r_{a}, r_{b}(a \in A, b \in B)$.

Элементы алгебры (1.14) (либо (1.15)) называются дифференциальными операторами типа $(A, B)$ в алгебре $A$. Соответственно алгебры $(1.14),(1.15)$ называются дифференциальными алгебрами типа $(A, B)$.

Элементы алгебры $\bar{B}^{\prime}$ (соответственно $B^{\prime}$ ) называются левоинвариантныци (правоинвариантными) дифференциальными операторами в алгебре $A$.

1.5. ОПРЕДЕЛЕНИЕ. Пусть $x \in A$, и пусть $l(x)$ (соответственно $r(x))$ - линейная оболочка элементов $x_{1}^{i}$ (соответственно $x_{2}^{i}$ ), определяемых разложением $\Delta x=x_{1} \otimes x_{2}$, при условии, что дополнительные элементы $x_{2}^{i}$ (соответственно $x_{1}^{i}$ ) линейно независимы. Подпространство $l(x)$ (соответственно $r(x))$ называется $л e-$ вым (правым) ростком әлемента $x$.

Заметим, что это определение корректно, поскольку можно считать, что элементы $x_{2}^{i}$ (соответственно $x_{1}^{i}$ ) составляют часть некоторого фиксированного базиса $F$ векторного пространства $A$. Очевидно, это определение не зависит от выбоpa $F$. Ясно также, что $\operatorname{dim} l(x), \operatorname{dim} r(x)<\infty$.

1.6. ПреДЛОЖенИЕ. Левыц̆ (правый) росток әлемента $x \in A$ есть циклический $\bar{B}^{\prime}{ }^{-м о д у л ь ~\left(B^{\prime}-м о д у л ь\right), ~ п о р о ж д е н н ы и ̆ ~ ә л е м е н т о м ~} x$ :

$$
\bar{B}^{\prime} x=l(x), \quad B^{\prime} x=r(x)
$$

для всех $x \in A$.

Действительно, если элементы $x_{1}^{i}(i=1, \ldots, n)$ линейно независимы, то существуют дуальные элементы $b_{i} \in B(i=1, \ldots, n)$, т.е. $b_{j}\left(x_{1}^{i}\right)=\delta_{i j}$ для всех $i, j=1, \ldots, n$. Поэтому из равенства (1.10) следует $B^{\prime} x=r(x)$. Аналогично, из равенства (1.11) следует $\bar{B}^{\prime} x=l(x)$. 


\section{§ 2. Теоремы плотности}

2.1. ПрЕДЛОЖЕНИЕ. Коммутант алгебры $D(A)$ в End $A$ скалярен, т.е. совпадает $c k \cdot 1$.

Действительно, если оператор $f \in$ End $A$ перестановочен со всеми $l_{a}(a \in A)$, то $f=r_{a}$ при $a=f(1)$. Перестановочность такого оператора со всеми $\partial=b^{\prime} \in B^{\prime}$ означает, что $\partial(x a)=\partial(x) a$ для всех $x \in A$. Полагая $x=1$, получаем

$$
\langle a, b\rangle=\langle\partial a, 1\rangle=\varepsilon(a)\langle 1, b\rangle
$$

для всех $b \in B$, т.е. $a=\varepsilon(a) 1$.

ЗАмЕчАниЕ. Очевидно, аналоги этого утверждения справедливы для всех дифференциальных алгебр (1.15).

2.2. Teорема. Пусть A есть алгебра Хопфа, и пусть $D(A)=\operatorname{Diff}(A, B)-$ дифференциальная алгебра, определенная в $n .1 .4$ (либо одна из алгебр (1.15)). Тогда имеем:

(i) $D(A)$-модуль $А$ абсолютно прост;

(ii) алгебра $D(A)$ плотна в End $A$ в алгебраическом смысле, а именно для каждого конечномерного подпространства $F \subset A$ имеем

$$
\left.D(A)\right|_{F}=\text { End }\left.A\right|_{F} .
$$

ЗАмЕчАНИЕ. Как известно из обшей теории модулей, утверждения (i), (ii) равносильны (см., например, [2]). Импликация (i) $\Rightarrow$ (ii) известна под названием теоремы плотности Джекобсона. Утверждение (ii) равносильно следующему:

(iii) для каждого набора линейно независимых әлементов $x_{i} \in A \quad(i=$ $1, \ldots, n)$ и каждого набора әлементов $y_{i} \in A(i=1, \ldots, n)$ существует оператор a $\in D(A)$ mакой, что $а x_{i}=y_{i}$ для всех $i=1, \ldots, n$.

Утверждение (ii) допускает следуюшую квантовомеханическую трактовку: каждая "наблюдаемая" $f \in$ End $A$ есть "функция" (в аппроксимативном смысле) от операторов "рождения" ( $a \in A)$ и “уничтожения" $\left(\partial \in B^{\prime}\right)$.

Доказательство излагается в п. 2.3 (см. также п. 2.4).

2.3. Пусть $0 \neq x_{0} \in A$. Применяя к элементу $x_{0}$ подходяший оператор $\partial=$ $b^{\prime} \in B^{\prime}$ и пользуясь соотношением $\varepsilon\left(\partial x_{0}\right)=\left\langle x_{0}, b\right\rangle$, находим, что в пространстве $r\left(x_{0}\right)$ содержится элемент $x=\partial x_{0}$, для которого $\varepsilon(x) \neq 0$. Изменяя нормировку, можем считать, что $\varepsilon(x)=1$. Но тогда из равенства (1.5) находим

$$
1=\gamma\left(x_{1}\right) x_{2} \in \operatorname{Ar}(x) \subset \operatorname{Ar}\left(x_{0}\right) .
$$

С другой стороны, используя (1.14), (1.16) (для алгебры $B^{\prime}$ ), получаем следуюшее общее правило:

$$
D(A) x=A r(x) .
$$


Применяя это правило к элементу $x_{0}$ и используя $(2.2)$, получаем $D(A) x_{0}=A$. Иначе говоря, каждый циклический подмодуль $D(A) x_{0}$ при $x_{0} \neq 0$ совпадает со всем пространством $A$. Отсюда заключаем, что $A$ есть простой $D(A)$-модуль.

Используя скалярность коммутанта $D(A)$ (предложение 2.1 ), получаем (i), откуда также следует (ii) (см. [2, теорема 3.6$]$ ).

2.4. ЗАмечаниЕ. Абсолютная простота $D(A)$-модуля $A$ может быть доказана непосредственно, если заметить, что все предыдущие рассуждения сохранятся при замене поля $k$ его произвольным расширением $\tilde{k}$. В частности, в п. 1.5 можно по-прежнему считать, что $F \subset A$, откуда следует аналог предложения 1.6 для алгебры $\widetilde{A}=A \otimes \tilde{k}$. Поэтому $D(\widetilde{A})$-модуль $\widetilde{A}$ прост (для всех $\tilde{k}$ ), т.е. $D(A)$-модуль $A$ абсолютно прост.

2.5. Существуют интересные примеры биалгебр (без антипода), для которых выполняется аналог теоремы плотности п. 2.2. Для изложения этих примеров нам понадобится ряд вспомогательных определений.

Пусть $A$ - ассоциативная алгебра с единицей, градуированная подпространствами $A_{\alpha}(\alpha \in \Gamma)$, где $\Gamma$ - некоторая аддитивная группа. Алгебра $A$ называется стандартной (или алгеброй полиномиального типа) [3], если выполняются следующие условия:

(a) $\Gamma$ - упорядоченная группа, порожденная системой $\pi$, состоящей из положительных образуюших $\alpha_{i}(i \in I)$;

(b) алгебра $A$ порождается однородными элементами $x_{i}(i \in I)$ со степенями однородности $\alpha_{i}=\operatorname{deg} x_{i}$.

Оператор $a \in$ End $A$ называется однородным степени $\alpha \in \Gamma$, если $a A_{\beta} \subset$ $A_{\alpha+\beta}$ для всех $\beta \in \Gamma$. Линейная оболочка $E(A)$ однородных операторов $a \in \operatorname{End} A$ есть градуированная алгебра (подалгебра в End $A$ ). Ясно также, что отображение $a \mapsto l_{a}$ (см. п. 1.3) определяет вложение градуированных алгебр $A \rightarrow E(A)$.

Мы будем рассматривать в $E(A)$ подкрученные коммутаторы $[., .]_{\tau}$, определяемые на однородных элементах $x, y \in E(A)$ по правилу

$$
[x, y]_{\tau}=x y-\tau(\alpha, \beta) y x
$$

где $\operatorname{deg} x=\alpha, \operatorname{deg} y=\beta, \tau: \Gamma \times \Gamma \rightarrow k-$ фиксированный бихарактер группы $\Gamma$. Заметим, что $\tau(\alpha, 0)=\tau(0, \beta)=1$ для всех $\alpha, \beta \in \Gamma$.

Условимся говорить, что $A$ есть алгебра типа $(\Gamma, \pi, \tau)$, если $A$ удовлетворяет условиям (a), (b) и в алгебре $E(A)$ фиксирован подкрученный коммутатор $[., .]_{\tau}$.

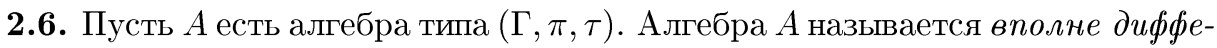
ренцируемой [3], если сушествуют операторы $\partial_{i} \in E(A)$, удовлетворяюшие соотношениям

$$
\left[\partial_{i}, x_{j}\right]_{\tau}=\delta_{i j}, \quad \partial_{i}(1)=0
$$

для всех $i, j \in I$. 
Легко проверить [3], что в этом случае в алгебре $A$ сушествует единственная невырожденная форма $(.,$.$) , удовлетворяющая условию нормировки (1,1)=1$ и условию контравариантности следующего вида:

$$
\left(x_{i} f, g\right)=\left(f, \partial_{i} g\right), \quad\left(\partial_{i} f, g\right)=\left(f, x_{i} g\right)
$$

для всех $f, g \in A$ и всех $i \in I$. Более того, форма $(.,$.$) симметрична, т.е.$ $(f, g)=(g, f)$ для всех $f, g \in A$. Форма $(.,$.$) называется канонической били-$ нейной формой в алгебре $A$.

Алгебра $A$ называется невырожденной [3], если каноническая форма ( . , . ) невырождена в $A$.

2.7. ПРЕДЛОЖЕНИЕ [3]. Для Каждой тройки $(\Gamma, \pi, \tau)$ (см. n. 2.5) существует единственная, с точностью до изоморфизма, невырожденная алгебра $A$ типа $(\Gamma, \pi, \tau)$. А именно

$$
A=F / N
$$

где $F$ - свободная алгебра типа $(\Gamma, \pi, \tau), N$ - ее идеал, определяемый как ядро билинейной формы (., .). Более того, алгебра (2.7) есть самодуальная биалгебра относительно формы $(.,$.$) .$

2.8. Легко проверить, что алгебра $D(A)$ для алгебры (2.7) порождается элементами $x_{i}, \partial_{i}(i \in I)$, и также

$$
D(A)=A D=D A,
$$

где $D=A^{\prime}$ - подалгебра с образуюшими $\partial_{i}(i \in I)$. Помимо этого алгебра $D(A)$ обладает следующей фильтрацией:

$$
D_{\alpha}(A)=\sum_{0 \leqslant \beta \leqslant \alpha} A D_{-\beta},
$$

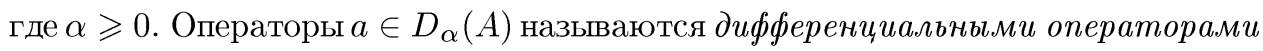
порядк $а \leqslant \alpha$.

2.9. Теорема. Пусть А есть невырожденная алгебра типа $(\Gamma, \pi, \tau)$. Тогда для каждого конечномерного подпространства $F \subset A$, порожденного әлементами степени $\leqslant \alpha$, имеем

$$
\left.D_{\alpha}(A)\right|_{F}=\text { End }\left.A\right|_{F} .
$$

Доказательство этой теоремы, изложенное в [3] для случая card $I<\infty$, переносится на общий случай без существенных изменений.

Таким образом, для алгебры (2.7) выполняется усиленный (градуированный) аналог теоремы плотности п. 2.2 .

2.10. ЗАмЕчАнИЕ. В [3] отмечен еще один вариант теоремы плотности для алгебры (2.7). А именно каждый оператор $f \in E(A)$ есть сумма ряда, сходящегося на элементах $x \in A$, с частичными суммами $f_{\alpha} \in D_{\alpha}(A)$.

Доказательство этого результата, изложенного в [3] для случая card $I<\infty$, также легко переносится на общий случай. 


\section{§ 3. Структурные теоремы}

3.1. Теорема. Пусть A, B - дуальная пара алгебр Хопфа. Тогда:

(i) отображсение $x \otimes y \mapsto x y$ определяет следующий изоморфизм векторных пространств:

$$
D(A)=A B^{\prime} \approx A \otimes B^{\prime}
$$

(ii) аналоги этого утверждения справедливы для всех дифференциальных алгебр (1.15);

(iii) если алгебра А коммутативна или кокоммутативна (т.е. В коммутативна), то алгебра $D(A)$ абсолютно проста.

Доказательство излагается в пп. 3.2-3.6.

3.2. ДоказатЕльство (i). Предположим, что в алгебре $D(A)$ выполняется следуюшее соотношение между элементами $a_{i} \in A, \partial_{i} \in B^{\prime}$ :

$$
\sum_{i=1}^{n} a_{i} \partial_{i}=0
$$

Условимся считать (не ограничивая общности), что элементы $a_{i}(i=1, \ldots, n)$ линейно независимы. Умножая (3.2) слева на $\bar{A}$, справа на $\bar{B}^{\prime}$ и используя перестановочность левых и правых умножений, получаем

$$
\sum_{i=1}^{n} a_{i} W \partial_{i}=0
$$

где $W=\bar{D}(\bar{A})$ в обозначениях (1.15). Применяя это равенство к элементу $x \in$ $A$, фиксируем базис $e_{j}(j=1, \ldots, m)$ в линейной оболочке элементов $\partial_{i} x \quad(i=$ $1, \ldots, n)$. Имеем

$$
\sum_{i=1}^{n} \sum_{j=1}^{m} a_{i} c_{i j} W e_{j}=0,
$$

где $c_{i j}$ - коэффициенты элемента $\partial_{i} x$ относительно базиса $e_{j}$. Используя теорему плотности п. 2.2 для алгебры $W$, находим элементы $w_{j} \in W(j=1, \ldots, m)$, удовлетворяющие соотношениям $w_{j}\left(e_{k}\right)=\delta_{j k}$ для всех $j, k=1, \ldots, m$. Подставляя эти элементы в (3.4), находим

$$
\sum_{i=1}^{n} a_{i} c_{i j}=0
$$

т.е. $c_{i j}=0$ для всех $i, j$. В результате $\partial_{i} x=0$ для всех $x$, т.е. $\partial_{i}=0$ для всех $i=1, \ldots, n$.

Полученный результат означает, что в алгебре $D(A)$ отсутствуют нетривиальные соотношения (3.2), что равносильно утверждению (i). Аналогично проверяется (ii). 
3.3. Пусть $A$ - алгебра Хопфа, $X$ - произвольный $A$-бимодуль. Как известно (см., например, [10]), операторы

$$
\pi(a) x=a_{1} x \tilde{a}_{2},
$$

где $a \in A, x \in X, \Delta a=a_{1} \otimes a_{2}, \tilde{a}=\gamma a$, определяют представление алгебры $A$ в пространстве $X$. Если $X$ есть алгебра, то

$$
\pi(a)(x y)=\pi\left(a_{1}\right) x \cdot \pi\left(a_{2}\right) y
$$

для всех $a \in A$ и всех $x, y \in X$.

3.4. Лемма. Пусть $\pi$ - представление алгебры $B^{\prime}$ в $D(A)$, определяемое по правилу (3.6) для әлементов $x \in D(A)$. Тогда для каждой пары $x \in A$, $\partial \in B^{\prime}$ оператор $\pi(\partial) x \in D(A)$ совпадает с оператором умножения на әлемент $\partial(x) \in A$.

Доказательство сводится к прямой проверке с использованием соотношений (1.12), (1.5), (1.4). Имеем

$$
[\pi(\partial) x] y=\partial_{1} x \tilde{\partial}_{2} y=\partial_{1}(x) \partial_{2} \tilde{\partial}_{3}(y)=\partial_{1}(x) \varepsilon\left(\partial_{2}\right) y=\partial(x) y
$$

для всех $y \in A$, где $\partial_{i}=b_{i}^{\prime}(i=1,2,3)$, при $\partial=b^{\prime} \in B, \tilde{\partial}=\tilde{b}^{\prime}, \Delta b=b_{1} \otimes b_{2}$, $\Delta^{2} b=b_{1} \otimes b_{2} \otimes b_{3}$.

Здесь мы используем стандартные правила сдвига индексов $(i \mapsto i \pm 1)$ при переходе от $\Delta b$ к $\Delta^{2} b$ (см., например, [10]).

3.5. Лемма. Пусть $I-$ - иеал алгебры $D(A)$, удовлетворяющий хотя бы одному из соотношений $I \cap A \neq 0, \quad I \cap B^{\prime} \neq 0$. Тогда имеем $I=D(A)$.

Действительно, пусть $0 \neq x \in I \cap A$. Используя инвариантность идеала $I$ относительно действия $\pi\left(B^{\prime}\right)$ и лемму п. 3.4 , получаем $B^{\prime}(x) \subset I$. Отсюда следует, что $I$ содержит $A B^{\prime}(x)=D(A)(x)=A$ (согласно теореме плотности п. 2.2). В результате $I=D(A)$.

Аналогично, если $I \cap B^{\prime} \neq 0$, то $I^{\prime} \cap B \neq 0$, откуда $I^{\prime}=D(B)$, т.е. $I=D(B)^{\prime}=$ $D(A)$.

3.6. ДоказАтельство(ii). Используя симметрию между $A$ и $B$, можем считать, не ограничивая общности, что алгебра $B$ коммутативна.

Пусть $I$ - идеал алгебры $D(A)$. Запишем произвольный элемент идеала $I$ в виде

$$
x=\sum_{i=1}^{n} a_{i} \partial_{i}
$$


где $a_{i} \in A, \partial_{i} \in B^{\prime}$ и сомножители $a_{i}(i=1, \ldots, n)$ линейно независимы. Применяя к $(3.8)$ преобразование $\pi\left(B^{\prime}\right)$ и используя коммутативность алгебры $B$, получаем следуюшее семейство элементов идеала $I$ :

$$
\pi(\partial) x=\sum_{i=1}^{n} \pi\left(\partial_{1}\right) a_{i} \cdot \pi\left(\partial_{2}\right) \partial_{i}=\sum_{i=1}^{n} \partial_{1}\left(a_{i}\right) \varepsilon\left(\partial_{2}\right) \partial_{i}=\sum_{i=1}^{n} \partial\left(a_{i}\right) \partial_{i}
$$

Здесь мы использовали лемму п. 3.4 и равенство $\pi(\partial) x=\varepsilon(\partial) x$ для элементов $\partial, x \in B^{\prime}$ (вытекающее из коммутативности алгебры $B$ ), где $\varepsilon(\partial)=\varepsilon(b)$ при $\partial=$ $b^{\prime} \in B^{\prime}$. Умножая полученное равенство слева на $A$, получаем

$$
\sum_{i=1}^{n} D(A)\left(a_{i}\right) \cdot \partial_{i} \subset I
$$

Применяя теорему плотности п. 2.2 для алгебры $D(A)$, находим $\partial_{i} \subset I$ для всех $i=1, \ldots, n$. Поэтому либо $I=0$, либо $I \cap B^{\prime} \neq 0$, т.е. $I=D(A)$ (лемма п. 3.5 ). Следовательно, алгебра $D(A)$ проста.

Остается заметить, что все приведенные рассуждения сохраняются при замене поля $k$ его произвольным расширением $\tilde{k}$ (см. п. 2.4). В результате получаем, что алгебра $D(A)$ абсолютно проста.

3.7. ЗАмечаниЕ. Пусть $A$-произвольная алгебра Хопфа. Повторяя рассуждения п. 3.6, находим, что $D(A)$ не содержит нетривиальных идеалов, инвариантных относительно действия $\pi\left(\bar{B}^{\prime}\right)$ в алгебре $D(A)$.

3.8. ПримеР. Пусть $\mathfrak{g}$ - симметризуемая алгебра Каца-Муди, и пусть $A=$ $A_{q}(\mathfrak{g}), B=U_{q}(\mathfrak{g})$ - классическая пара алгебр Хопфа, ассоциированных с $\mathfrak{g}$ (см. [7], [10]). Напомним, что $B$ есть квантовая оболочка Дринфельда-Джимбо алгебры $\mathfrak{g}$, $A=B^{+}$- координатная алгебра, ассоциированная с алгеброй $\mathfrak{g}$. Заметим, что алгебра $B$ изоморфна $B^{\prime}$ (относительно инволюции Шевалле в алгебре $\mathfrak{g}$ ). Поэтому, используя теорему $3.1,(\mathrm{i})$, получаем

$$
D(A) \approx A_{q}(\mathfrak{g}) \otimes U_{q}(\mathfrak{g}) .
$$

Заметим, что для алгебр конечного типа $(\operatorname{dim} \mathfrak{g}<\infty)$ координатная алгебра $A_{q}(\mathfrak{g})$ иногда обозначается $F_{q}(G)$, где $G$ - алгебраическая группа, для которой $\mathfrak{g}=\operatorname{Lie} G$ (см., например, [12]).

3.9. ОПреДЕлЕниЕ. Пусть $A, B$ - дуальная пара биалгебр, и пусть $W(A)=$ $W(A, B)$ - ассоциативная алгебра с единицей, порожденная элементами $A \cup B^{\prime}$ и определяюшими соотношениями следуюшего вида:

$$
\begin{array}{ll}
x y=\mu(x, y), & x, y \in A \text { или } x, y \in B^{\prime}, \\
\partial x=\partial_{1}(x) \partial_{2}, & x \in A, \quad \partial \in B^{\prime},
\end{array}
$$


где $\mu$ означает операцию умножения в алгебрах $A, B^{\prime}$. Условимся говорить, что $W(A)$ есть алгебра Вейля, ассоциированная с парой $A, B$.

Согласно (1.13) алгебра Вейля $W(A)$ действует в $A$ посредством гомоморфизма $\varphi: W(A) \rightarrow D(A)$, определяемого на элементах $x \in A \cup B^{\prime}$ по правилу $\varphi(x)=x$. Более того, $W(A)$ накрывает $D(A)$, т.е.

$$
D(A) \approx W(A) / \operatorname{ker} \varphi
$$

3.10. Теорема. Пусть $W(A)=W(A, B)$ - алгебра Вейля, определенная $\boldsymbol{в}$ n. 3.9. Тогда:

(i) каждая из алгебр $A, B^{\prime}$ изоморфна одноименной подалгебре в $W(A)$;

(ii) отображение $x \otimes y \mapsto x y$ определяет следующий изоморфизм векторных пространств:

$$
W(A)=A B^{\prime} \approx A \otimes B^{\prime}
$$

(iii) если $A$ - алгебра Хопфа, то $\operatorname{ker} \varphi=0$, т.е. отображение $\varphi$ определяет изоморфизм алгебр $W(A), D(A)$.

Действительно, пусть $\alpha$ - гомоморфизм накрытия $F \rightarrow W(A)$, где $F$ - свободная алгебра, порожденная множеством $A \cup B^{\prime}$. Тогда $\varphi \circ \alpha=1$ на $A \cup B^{\prime}$, откуда следует (i) (т.е. $\alpha$ есть вложение для пары $A, B^{\prime}$ ).

Для доказательства (ii) заметим, что любой одночлен от элементов $A \cup B^{\prime}$ приводится к виду $A B^{\prime}$ посредством тождеств (3.10). Покажем, что это приведение единственно, откуда следует сушествование изоморфизма в (3.12). Воспользуемся следуюшими соотношениями коммутации в алгебре $W(A)$ :

$$
\begin{aligned}
& \partial x y=\partial_{1}(x y) \partial_{2}=\partial_{1}(x) \partial_{2}(y) \partial_{3} \\
& \partial \delta x=\partial \delta_{1}(x) \delta_{2}=\partial_{1} \delta_{1}(x) \partial_{2} \delta_{2}
\end{aligned}
$$

в обозначениях п. 3.4, где $x, y \in A$ и $\partial, \delta \in B^{\prime}$. Соотношение (3.13) позволяет заключить, что перестановка элемента $\partial$ с произведением $x y$ приводит к тому же результату, что и его последовательная перестановка с элементами $x, y$. Аналогично интерпретируется (3.14). Отсюда следует (ii).

Утверждение (iii) вытекает теперь из теоремы п. 3.1.

3.11. ЗАмечаниЕ. Утверждение (ii) выполняется также для биалгебр $A=$ $F / N$, определенных в п. 2.7. Более того, в этом случае имеем (см. [3])

$$
D(A) \approx A \otimes D \approx D \otimes A .
$$




\section{§ 4. Алгебра $Q(A)$}

4.1. Пусть $A$ - алгебра Хопфа. Операторы $\pi(x)=\operatorname{ad}_{x}$ определяют $n р и с о е д u$ ненное действие алгебры $A$ в пространстве $A$ :

$$
\operatorname{ad}_{x} y=x_{1} y \tilde{x}_{2},
$$

где $x, y \in A, \Delta x=x_{1} \otimes x_{2}, \tilde{x}=\gamma x$. С другой стороны, определим присоединенное кодействие $\mathrm{Ad}: A \rightarrow A \otimes A$ следуюшим образом:

$$
\operatorname{Ad} x=x_{1} \tilde{x}_{3} \otimes x_{2}
$$

где $\Delta^{2} x=x_{1} \otimes x_{2} \otimes x_{3}$. Отображение (4.2) порождает следующее семейство линейньх операторов в алгебре $A$ :

$$
\operatorname{Ad}_{f} x=f\left(x_{1} \tilde{x}_{3}\right) x_{2}
$$

для всех $f \in A^{*}$.

Операторы (4.1)-(4.3) связаны между собой известными соотношениями двойственности. Мы приводим эти соотношения для полноты изложения.

4.2. Лемма. Пусть $A, B$ - дуальная пара алгебр Хопфа. Тогда:

(i) отображение $\mathrm{ad}: A \otimes A \rightarrow A$, определяемое по правилу $(x, y) \mapsto \operatorname{ad}_{x} y$, сопряжено отображению $\mathrm{Ad}: A \rightarrow A \otimes A$, т.е.

$$
\left\langle\operatorname{ad}_{x} y, f\right\rangle=\langle x \otimes y, f\rangle
$$

для всех $x, y \in A$ u всех $f \in B$;

(ii) отображение $\operatorname{Ad}_{f}: A \rightarrow A$ сопряжено присоединенному действию $\operatorname{ad}_{f}: B \rightarrow B$ алгебры $B$, m.e.

$$
\left\langle\operatorname{Ad}_{f} x, g\right\rangle=\left\langle x, \operatorname{ad}_{f} g\right\rangle
$$

для всех $x \in A$ и всех $f, g \in B$;

(iii) подпространство $L \subset A$ инвариантно относительно операторов (4.3) при $f \in B$ тогда и только тогда, когда $\operatorname{Ad} L \subset A \otimes L$.

ДоКАЗАТЕЛЬСТво сводится к непосредственной проверке. Полагая $\Delta^{2} f=$ $f_{1} \otimes f_{2} \otimes f_{3}$, получаем

$$
\left\langle\operatorname{ad}_{x} y, f\right\rangle=f_{1}\left(x_{1}\right) f_{2}(y) f_{3}\left(\tilde{x}_{2}\right)=f_{1} \tilde{f}_{3}(x) f_{2}(y)=\langle x \otimes y, \operatorname{Ad} f\rangle .
$$

Отсюда (изменяя роли $A, B$ ) получаем

$$
\left\langle\operatorname{Ad}_{f} x, g\right\rangle=f\left(x_{1} \tilde{x}_{3}\right) g\left(x_{2}\right)=\langle\operatorname{Ad} x, f \otimes g\rangle=\left\langle x, \operatorname{ad}_{f} g\right\rangle .
$$


Ясно также, что включение $\operatorname{Ad} L \subset A \otimes L$ влечет инвариантность $L$ относительно операторов (4.3) для всех $f \in A^{*}$. Обратно, пусть $\operatorname{Ad}_{f} L \subset L$ для всех $f \in B$. Иначе говоря, для всякого элемента $z \in \operatorname{Ad} L$, представленного в виде суммы элементов $x_{i} \otimes y_{i}(i=1, \ldots, n)$, имеем

$$
\sum_{i=1}^{n} f\left(x_{i}\right) y_{i} \in L
$$

для всех $f \in B$. Условимся считать, что элементы $x_{i}(i=1, \ldots, n)$, входящие в это выражение, линейно независимы, и пусть $f_{i}(i=1, \ldots, n)$ - дуальная система в $B$, т.е. $f_{i}\left(x_{j}\right)=\delta_{i j}$ для всех $i, j=1, \ldots, n$. Подставляя эти элементы в (4.6), находим $y_{i} \in L$ для всех $i=1, \ldots, n$, т.е. $z \in A \otimes L$.

4.3. ОПРЕДЕЛЕНИЕ. Пусть $A, B$ - дуальная пара алгебр Хопфа, и пусть $Q(A, B)$ - ассоциативная алгебра с единицей, порожденная элементами $A \cup B$ и определяюшими соотношениями следуюшего вида:

$$
\begin{array}{llrl}
x y & =\mu(x, y), & x, y \in A \text { или } x, y \in B, \\
f x=f_{1}\left(x_{3}\right) f_{3}\left(\tilde{x}_{1}\right) x_{2} f_{2}, & x \in A, \quad f \in B,
\end{array}
$$

где использованы обозначения п. 4.2 , т.е. $\Delta^{2} x=x_{1} \otimes x_{2} \otimes x_{3}, \tilde{x}=\gamma x, \Delta^{2} f=f_{1} \otimes$ $f_{2} \otimes f_{3}$. Алгебра $Q(A, B)$ называется квантовым дублем Дринфельда, ассоциированным с парой $A, B$.

ЗАмечАниЕ. Существуют известные модификации в определении квантового дубля Дринфельда (см., например, [7], [9], [10]). Мы используем определение, согласованное с операторами (4.3), а также нестандартное обозначение $Q(A, B)$ (вместо $D(A, B), \mathscr{D}(A, B)$ и т.д.) во избежание возможного смешения с обозначением дифференциальных алгебр, введенных в п. 1.4.

Как известно, алгебра $Q(A, B)$ обладает структурой алгебры Хопфа, согласованной с исходными структурами в алгебрах $A, B$. Мы не станем приводить эти определения, поскольку в дальнейшем они не используются.

4.4. Теорема. Пусть $Q(A)=Q(A, B)$ - квантовый дубль Дринфельда, определенный в n. 4.3. Тогда:

(i) алгебра $Q(A)$ действует в пространстве А посредством операторов

$$
a \cdot x=a x, \quad f \cdot x=\operatorname{Ad}_{\tilde{f}} x,
$$

где $a, x \in A, \quad f \in B$;

(ii) отображение $x \otimes y \mapsto x y$ определяет следующие изоморфизмы векторных пространств:

$$
Q(A)=A B \approx A \otimes B, \quad Q(A)=B A \approx B \otimes A,
$$


где исходные алгебры $A, B$ отождествляются (соответственно) с подалгебрами $A \otimes 1, \quad 1 \otimes B$ и т.д.

Заметим вначале, что $f \mapsto \operatorname{Ad}_{f}$ есть антипредставление алгебры $B$ (см. (4.5)). Поэтому формулы (4.9) определяют представление алгебр $A, B$ в пространстве $A$. Теперь для доказательства (i) достаточно проверить, что тождество (4.8) сохраняется при переходе к операторам (4.9), т.е. $f \cdot a x=f a \cdot x$ для всех $a, x \in A$ и всех $f \in B$. Действительно, имеем

$$
\begin{aligned}
f \cdot a x & =f\left(a_{3} x_{3} \tilde{x}_{1} \tilde{a}_{1}\right) a_{2} x_{2}=f_{1}\left(a_{3}\right) f_{3}\left(\tilde{a}_{1}\right) f_{2}\left(x_{3} \tilde{x}_{1}\right) a_{2} x_{2} \\
& =f_{1}\left(a_{3}\right) f_{3}\left(\tilde{a}_{1}\right) a_{2} f_{2} \cdot x=f a \cdot x .
\end{aligned}
$$

Доказательство (ii) аналогично доказательству теоремы п. 3.11. Детали рассуждения можно найти в [7], [9], [10].

4.5. СлЕДСтвиЕ. Пусть $N$-ядро присоединенного представления $f \mapsto \operatorname{ad}_{f}$ алгебры $B$, и пусть

$$
\widetilde{Q}(A)=Q(A) / N
$$

где $N$ отождествляется $c 1 \otimes N$ в алгебре $Q(A) \approx A \otimes B$. Тогда операторы (4.9) определяют в А структуру $\widetilde{Q}(A)$-модуля.

Соответственно $B$ наделяется структурой сопряженного $Q(A)$-модуля $(\widetilde{Q}(A)$ модуля). Согласно п. 4.2 этот модуль порождается операторами $a^{\prime}(a \in A), \operatorname{ad}_{f}$ $(f \in B)$.

ЗАмечАниЕ. Можно показать, по аналогии с п. 3.11 , что $A$ есть точньй $\widetilde{Q}(A)$ модуль, т.е. операторы (4.9) определяют вложение $\widetilde{Q}(A)$ в алгебру End $A$.

4.6. Условимся о некоторых обозначениях, используемых в дальнейшем. Мы будем рассматривать $A^{*}$ как $A$-бимодуль с операторами

$$
(a * f)(x)=f(x a), \quad(f * a)(x)=f(a x),
$$

где $a, x \in A, f \in A^{*}$.

Для каждого подпространства $L \subset A$ пусть $L^{0}$ - его аннулятор в $A^{*}$ (т.е. множество всех функционалов $f \in A^{*}$, равных нулю на $L$ ). Очевидно, элементы $f \in L^{0}$ допускают интерпретацию как линейные функционалы в $A / L$. При этом $L^{0}$ отождествляется с пространством, сопряженным к $A / L$.

Ясно также, что отображение $L \mapsto L^{0}$ переводит каждый левый (правый) идеал $L$ алгебры $A$ в правый (левый) подмодуль $L^{0} \subset A^{*}$.

Очевидно, $L \subset L^{00}$. Если $\operatorname{codim} L<\infty$, то $L=L^{00}$. Отсюда заключаем, что отображение $L \mapsto L^{0}$ определяет биекцию между левыми (правыми) идеалами $L \subset A$ конечной коразмерности и правыми (левыми) конечномерными подмодулями $L^{0} \subset A^{*}$. 


\section{§ 5. Дифференциальные формы}

5.1. В этом параграфе излагается обшая схема дифференциального исчисления над алгеброй Хопфа $A$, в известном смысле двойственная к предложенной в [15]. В отличие от классического случая (многообразия, группы Ли) эта схема не единственна и связана с выбором специальных левых (правых) идеалов алгебры $A$.

Пусть $L \subset \operatorname{ker} \varepsilon-$ левый идеал алгебры $A$, и пусть $L_{1}=k \cdot 1 \oplus L$. Тогда имеем

$$
A / L=k \cdot 1 \oplus A / L_{1}
$$

где единичный элемент $1 \in A$ отождествляется со своим образом в $A / L$. Соответствующие сопряженные пространства (аннуляторы) $L^{0}, L_{1}^{0}$ связаны соотношением

$$
L^{0}=k \cdot \varepsilon \oplus L_{1}^{0}
$$

Фиксируем базис $a_{i}(i \in I)$ векторного пространства $A / L_{1}$, и пусть $b_{i}(i \in I)$ соответствующая координатная система в $L_{1}^{0}$, так что

$$
x=\sum_{i} b_{i}(x) a_{i}
$$

для каждого $x \in A \bmod L_{1}$. Очевидно, $a_{i}, b_{i}(i \in I)$ суть дуальные системы, т.е. $b_{i}\left(a_{j}\right)=\delta_{i j}$ для всех $i, j \in I$.

Пусть $F$ - линейная оболочка системы $b_{i}(i \in I)$, и пусть $F_{1}=k \cdot \varepsilon \oplus F$. Нетрудно видеть (см. п. 5.2), что $F_{1}$ есть (правый) $A$-модуль относительно действия (4.16). Условимся говорить, что $F_{1}$ есть касательный $A$-модуль, определяемый идеалом $L$ и базисом $a_{i}(i \in I)$.

ЗАмечАниЕ. Если $\operatorname{codim} L<\infty$, то имеем $F_{1}=L^{0}$, откуда следует, что в этом случае модуль $F_{1}$ не зависит от выбора базиса $a_{i}(i \in I)$.

5.2. Условимся отождествлять элементы $a_{i}(i \in I)$ с их фиксированными представителями в $\operatorname{ker} \varepsilon$. Разлагая элемент $y \in A \bmod L$ по базису $1, a_{i}(i \in I)$ с коэффициентами $\varepsilon, b_{i}(i \in I)$ и используя включение $x L \subset L$ при $x \in A$, находим

$$
b_{i}(x y)=f_{i}(x) \varepsilon(y)+\sum_{j} f_{i j}(x) b_{j}(y)
$$

для всех $x, y \in A$ с коэффициентами $f_{i}, f_{i j} \in A^{*}$. Полагая $y=1$, получаем $f_{i}=b_{i}$. Полагая $y=a_{j}$, получаем

$$
f_{i j}(x)=b_{i}\left(x a_{j}\right)
$$

для всех $x \in A$ и всех $i, j \in I$. Используя закон ассоциативности алгебры $A$ и равенство (5.4), находим

$$
f_{i j}(x y)=\sum_{k} f_{i k}(x) f_{k j}(y)
$$


для всех $x, y \in A$ и всех $i, j \in I$. Ясно также, что $f_{i j}(1)=\delta_{i j}$ для всех $i, j \in I$.

Заметим, что система $b_{i}(i \in I)$ локально конечна, т.е. удовлетворяет условию

$(\alpha)$ для каждого $x \in A$ лишь конечное число значений $b_{i}(x)$ отлично от нуля.

Отсюда следует, согласно (5.5), что система $f=\left(f_{i j}\right)$ локально конечна по столбцам, т.е. удовлетворяет условию

( $\beta$ ) для каждой пары $x \in A, j \in I$ лишь конечное число значений $f_{i j}(x)$ отлично от нуля.

Элемент $b_{i}$ содержится в $A^{+}$(см. п. 1.2 ) тогда и только тогда, когда вьполняется более сильное условие конечности для матрицы $f$ :

$\left(\gamma_{i}\right)$ лишь конечное число функционалов $f_{i j}(j \in I)$ отлично от нуля.

Очевидно, в этом случае соотношение (5.4) принимает следующий вид:

$$
\Delta b_{i}=b_{i} \otimes 1+\sum_{j} f_{i j} \otimes b_{j}
$$

где $1 \in A^{+}$совпадает с коединицей алгебры $A$ (см. п. 1.2).

5.4. Для каждого $f \in A^{*}$ мы будем рассматривать соответствуюшие левоинвариантные (правоинвариантные) векторные поля $\partial(x)=f * x$ (соответственно $\bar{\partial}(x)=x * f)$, т.е.

$$
\partial(x)=x_{1} f\left(x_{2}\right), \quad \bar{\partial}(x)=f\left(x_{1}\right) x_{2}
$$

в обозначениях п. 1.3. Заметим, что $f \in A^{*}$ восстанавливается по векторным полям (4.8) по правилу $f=\varepsilon \circ \partial=\varepsilon \circ \bar{\partial}$, т.е.

$$
f(x)=\varepsilon(\partial(x))=\varepsilon(\bar{\partial}(x))
$$

для всех $x \in A$.

Применяя (5.4), получаем следующие правила Лейбница для векторных полей $\partial_{i}(x)=b_{i} * x:$

$$
\partial_{i}(x y)=\partial_{i}(x) y+\sum_{j} c_{i j}(x) \partial_{j}(y)
$$

с коэффициентами $c_{i j}(x)=f_{i j} * x$. Аналогично, из равенства (5.6) следует

$$
c_{i j}(x y)=\sum_{k} c_{i k}(x) c_{k j}(y)
$$

для всех $x, y \in A$ и всех $i, j \in I$. Ясно также, что $\partial_{i}(1)=0, c_{i j}(1)=\delta_{i j}$ для всех $i, j \in I$.

Соотношения (5.10) выполняются также при замене $\partial_{i}(x)$ на $\bar{\partial}_{i}(x)=x * b_{i} \mathrm{c}$ коэффициентами $\bar{c}_{i j}(x)=x * f_{i j}$.

ЗАмечание. Аналогично можно было бы рассматривать правые идеалы $R \subset$ $\operatorname{ker} \varepsilon$ алгебры $A$. Однако равенство $L=\gamma R$ устанавливает биекцию между левыми и правыми идеалами алгебры $A$. 
5.5. ОПРЕДЕЛЕНИЕ. Пусть $\Gamma=\Gamma(L)$ - свободный правый $A$-модуль с образуюшими $\omega_{i}(i \in I)$, т.е. множество всех элементов вида

$$
\omega(x)=\sum_{i} \omega_{i} x_{i}
$$

с коэффициентами $x_{i} \in A$ и покомпонентными операциями $x_{i} \mapsto x_{i} a(a \in A)$. Полагая

$$
x \omega_{j}=\sum_{i} \omega_{i} c_{i j}(x)
$$

в обозначениях п. 4.4 , мы наделяем $\Gamma$ структурой левого $A$-модуля. Таким образом, Г наделяется структурой $A$-бимодуля.

Определим отображение $d: A \rightarrow \Gamma$, полагая

$$
d x=\sum_{i} \omega_{i} \partial_{i}(x)
$$

где $\partial_{i}(i \in I)$ - векторные поля, определенные в п. 5.4. Используя (5.9), (5.12), находим

$$
d(x y)=d x \cdot y+x \cdot d y
$$

для всех $x, y \in A$.

Элементы бимодуля Г назьваются левыми дифференциальными формами (первого порядка) над алгеброй $A$. Оператор $d: A \rightarrow \Gamma$ называется левым nолным.м дифференииалом в алгебре $A$.

Аналогично, с заменой $\partial_{i}, c_{i j}$ на $\bar{\partial}_{i}, \bar{c}_{i j}$ определяется бимодуль $\bar{\Gamma}=\bar{\Gamma}(L)$, состояший из правых дифференциальных форм над алгеброй $A$.

ЗАмечАниЕ. Элементы $\omega_{i}(i \in I)$ преобразуются ковариантно базисными элементами $a_{i}(i \in I)$ относительно перевыбора базиса в $A / L$ (см. п. 5.1). В этом смысле определение $\Gamma(L)$ не зависит от выбора базиса в $A / L$.

5.6. ЛЕмма. Положим $\tilde{b}_{i}=b_{i} \circ \gamma, \tilde{f}_{i j}=f_{i j} \circ \gamma$, u nусть $\tilde{\partial}_{i}(x)=\tilde{b}_{i} * x$, $\tilde{c}_{i j}(x)=\tilde{f}_{i j} * x-$ соответствующие левоинвариантные векторные поля. Тогда:

(i) операторные матрицы $c=\left(c_{i j}\right), \tilde{c}=\left(\tilde{c}_{i j}\right)$ удовлетворяют соотношениям $\tilde{c} c=c \tilde{c}=1$, m.e.

$$
\sum_{k} \tilde{c}_{i k} c_{k j}=\sum_{k} c_{i k} \tilde{c}_{k j}=\delta_{i j}
$$

для всех $i, j \in I$;

(ii) операторы $\partial_{i}, \tilde{\partial}_{i}$ удовлетворяют соотношениям

$$
\partial_{i}=-\sum_{j} c_{i j} \tilde{\partial}_{j}, \quad \tilde{\partial}_{i}=-\sum_{j} \tilde{c}_{i j} \partial_{j}
$$

для всех $i \in I$. 
Доказательство сводится к прямой проверке (на элементах $x \in A$ ). Например, используя (5.10), получаем

$$
\begin{aligned}
\sum_{k} \tilde{c}_{i k} c_{k j}(x) & =\sum_{k} \tilde{c}_{i k}\left[x_{1} f_{k j}\left(x_{2}\right)\right]=x_{1} \sum_{k} f_{i k}\left(\tilde{x}_{2}\right) f_{k j}\left(x_{3}\right) \\
& =x_{1} f_{i j}\left(\varepsilon\left(x_{2}\right)\right)=\delta_{i j} x_{1} \varepsilon\left(x_{2}\right)=\delta_{i j} x
\end{aligned}
$$

где положено $\Delta x=x_{1} \otimes x_{2}, \Delta^{2} x=x_{1} \otimes x_{2} \otimes x_{3}$. Аналогично, используя (5.9), получаем

$$
\begin{aligned}
\sum_{j} \tilde{c}_{i j} \partial_{j}(x) & =\sum_{j} \tilde{c}_{i j}\left[x_{1} b_{j}\left(x_{2}\right)\right]=x_{1} \sum_{j} f_{i j}\left(\tilde{x}_{2}\right) b_{j}\left(x_{3}\right) \\
& =x_{1}\left[b_{i}\left(\varepsilon\left(x_{2}\right)\right)-b_{i}\left(\tilde{x}_{2}\right)\right]=-\tilde{\partial}_{i}(x) .
\end{aligned}
$$

Точно так же проверяются остальные соотношения (5.15), (5.16).

5.7. ПРЕДЛОЖЕНИЕ. Элементы (5.11) однозначно записываются в следующем виде:

$$
\omega(x)=\sum_{i} \bar{x}_{i} \omega_{i}
$$

с коэффициентами $\bar{x}_{i}=\sum_{j} \tilde{c}_{i j}\left(x_{j}\right)$. В частности, Г есть свободный левый А-модуль. При этом

$$
d x=-\sum_{i} \tilde{\partial}_{i}(x) \omega_{i}
$$

Доказательство сводится к элементарной проверке с использованием леммы п. 5.6 .

5.8. ПРЕДЛОЖЕНИЕ. Элементы (5.11) могут быть записаны в виде суммы әлементов вида $d x_{k} \cdot y_{k}\left(\right.$ либо $\left.x_{k} \cdot d y_{k}\right)$, где $x_{k}, y_{k} \in A, k=1, \ldots, n$.

Достаточно рассматривать базисные элементы $\omega_{i}(i \in I)$. Например, для модуля $\bar{\Gamma}=\bar{\Gamma}(L)$ имеем

$$
\begin{aligned}
\omega_{j} & =\sum_{i} \omega_{i} b_{i}\left(a_{j}\right)=\sum_{i} \omega_{i} b_{i}\left(a_{j 1}\right) \varepsilon\left(a_{j 2}\right) \\
& =\sum_{i} \omega_{i} b_{i}\left(a_{j 1}\right) a_{j 2} \tilde{a}_{j 3}=\sum_{j} \omega_{i} \partial_{i}\left(a_{j 1}\right) \tilde{a}_{j 2}=d a_{j 1} \cdot \tilde{a}_{j 2},
\end{aligned}
$$

где $\tilde{x}=\gamma x$. Аналогично, $\omega_{j}=\tilde{a}_{j 1} \cdot d a_{j 2}$, где $\tilde{x}=\gamma^{-1} x$. Случай $\Gamma=\Gamma(L)$ рассматривается аналогично с использованием (5.18). 


\section{§ 6. Элементы геометрии}

6.1. Напомним основные определения [15], связанные с дифференциальным исчислением (первого порядка) над алгеброй Хопфа $A$.

Бимодуль Г над алгеброй $A$ называется дифференциальным исчислением первого порядка, если он снабжен оператором $d: A \rightarrow \Gamma$ (полньй дифференциал), удовлетворяюшим соотношению (5.14), и если элементы $\omega \in \Gamma$ допускают представление в виде

$$
\omega=\sum_{k=1}^{n} x_{k} d y_{k}
$$

где $x_{k}, y_{k} \in A(k=1, \ldots, n)$.

Бимодуль Г называется левоковариантным, если он снабжен оператором $\delta$ : $\Gamma \rightarrow A \otimes \Gamma$, удовлетворяюшим соотношению

$$
\delta(\omega)=\sum_{k=1}^{n} \Delta x_{k}(1 \otimes d) \Delta y_{k}
$$

для элементов вида (6.1). Как показано в [15], оператор $\delta$ удовлетворяет также следующим соотношениям:

$$
\begin{gathered}
\delta(a \omega b)=\Delta(a) \delta(\omega) \Delta(b), \\
(\Delta \otimes 1) \delta=(1 \otimes \delta) \delta, \quad(\varepsilon \otimes 1) \delta=1, \\
\delta d=(1 \otimes d) \delta,
\end{gathered}
$$

где $a, b \in A, \omega \in \Gamma$.

Бимодуль $A$ называется правоковариантныц, если он снабжен оператором $\delta^{\prime}: \Gamma \rightarrow \Gamma \otimes A$, удовлетворяющим соотношению

$$
\delta^{\prime}(\omega)=\sum_{k=1}^{n} \Delta x_{k}(d \otimes 1) \Delta y_{k}
$$

для элементов вида (6.1). В этом случае для оператора $\delta^{\prime}$ также выполняются аналоги $(6.3),(6.4),(6.6)$ с заменой $\Delta \otimes 1$ на $1 \otimes \Delta$ и т.д.

Бимодуль $Г$ называется биковариантным, если он одновременно левоинвариантен и правоинвариантен. Как показано в [15], операторы $\delta, \delta^{\prime}$ в этом случае связаны следующим соотношением согласованности:

$$
(\delta \otimes 1) \delta^{\prime}=\left(1 \otimes \delta^{\prime}\right) \delta .
$$

Элемент $\omega \in$ Г называется левоинвариантныцм (правоинвариантным), если $\delta \omega=1 \otimes \omega\left(\right.$ соответственно $\left.\delta^{\prime} \omega=\omega \otimes 1\right)$. Подпространство левых (правых) инвариантов бимодуля $Г$ обозначается inv $\Gamma$ (соответственно $\left.\Gamma_{\text {inv }}\right)$.

Условимся также говорить, что $(\Gamma, d)$ есть левоковариантное (правоковариантное) исчисление конечного ранга, если $\operatorname{dim}\left({ }_{\mathrm{inv}} \Gamma\right)<\infty$ (соответственно $\left.\operatorname{dim}\left(\Gamma_{\text {inv }}\right)<\infty\right)$. 
6.2. ПРЕДЛОЖЕНИЕ. Дифференциальное исчисление Г $L$ ) (соответственно $\bar{\Gamma}(L)$ ), определенное в $n .5 .5$, левоковариантно (правоковариантно).

Действительно, определим отображение $\delta: \Gamma(L) \rightarrow A \otimes \Gamma(L)$ по правилу $\delta \omega_{i}=$ $1 \otimes \omega_{i}$ с продолжением на весь бимодуль $\Gamma(L)$ по правилу (6.3). Легко проверить (см. п. 7.5), что $\Delta \circ \partial=(1 \otimes \partial) \circ \Delta$ для каждого левоинвариантного векторного поля $\partial(x)=f * x\left(f \in A^{*}\right)$. Отсюда следует (6.2), т.е. бимодуль $\Gamma(L)$ левоковариантен. Случай $\bar{\Gamma}(L)$ рассматривается аналогично (с определением $\left.\delta^{\prime} \omega_{i}=\omega_{i} \otimes 1\right)$.

6.3. ТеОРема. Пусть $\Gamma(L), \bar{\Gamma}(L)$ - дифференциальные исчисления, определенные в n. 5.5. Тогда:

(i) каждое левоковариантное (правоковариантное) дифференциальное исчисление первого порядка над алгеброй А совпадает с одним из $\Gamma(L)$ (coответственно $\bar{\Gamma}(L))$, где $L \subset \operatorname{ker} \varepsilon-$ левьй идеал алгебры $A$;

(ii) дифференциальное исчисление $\Gamma(L)$ (аналогично $\bar{\Gamma}(L))$ биковариантно тогда и только тогда, когда идеал $L$ Ad-инвариантен относительно действия (4.2).

Действительно, (i) вытекает непосредственно из теоремы п. 2.1 (соответственно п. 2.3) статьи [15], где показано, что каждое левоковариантное (правоковариантное) дифференциальное исчисление $(\Gamma, d)$ есть левый (правый) свободньй $A$-модуль, базис которого определяется как произвольный базис векторного пространства inv $\Gamma$ (соответственно $\Gamma_{\text {inv }}$ ). При этом соотношения Лейбница (5.9) возникают как следствия структурных соотношений (5.12), (5.14), определенных в $(\Gamma, d)$.

Заметим, что условия локальной конечности $(\alpha),(\beta)$ (см. п. 5.2) содержатся (явно или неявно) в конструкции [15]. А именно условие $(\alpha)$ (соответственно $(\beta)$ ) следует из локальной конечности разложений (5.13) (соответственно (5.12)), определенных в $(\Gamma, d)$.

Помимо этого в $[15]$ показано (теоремы $1.5,1.6$ ), что каждое левоковариантное (правоковариантное) дифференциальное исчисление $(\Gamma, d)$ однозначно характеризуется некоторым правым идеалом $R \subset \operatorname{ker} \varepsilon$. Легко проверить, что $R=\gamma^{-1} L$ при $\Gamma=\Gamma(L)$. Согласно [15, теорема 1.8$]$ бимодуль $\Gamma$ биковариантен тогда и только тогда, когда идеал $R$ ad-инвариантен в смысле [15], т.е. идеал $L \mathrm{Ad}$-инвариантен относительно действия (4.2). Отсюда следует (ii).

6.4. СледСтвиЕ. Отображение $L \mapsto \Gamma(L)$ (соответственно $L \mapsto \bar{\Gamma}(L)$ ) определяет сюрбекиию множества конечномерных касательных $A$-модулей $F_{1}=L^{0}$ на множество левоковариантных (правоковариантных) дифференииальных исчислений конечного ранга над алгеброй $A$.

ЗАмечаниЕ. Отображение $L \mapsto \Gamma(L)$ можно рассматривать как биекцию на семейство дифференциальных исчислений, определенных в [15]. Однако остается открытым вопрос о совпадении (изоморфизме) дифференциальных исчислений $\Gamma\left(L_{1}\right), \Gamma\left(L_{2}\right)$ при различных $L_{1}, L_{2}$. 
6.5. Теорема. Дифференциальное исчисление $\Gamma(L)$ (аналогично $\bar{\Gamma}(L))$ биковариантно тогда и только тогда, когда L есть подпространство в $\operatorname{ker} \varepsilon$, инвариантное относительно действия квантового дубля $Q(A, B)$, где $B$ произвольная дуальная биалгебра алгебры $А$.

Действительно, $Q(A, B)$-инвариантность сводится к одновременной $A$-инвариантности и $B$-инвариантности, причем $A$-инвариантность означает, что $L$ есть левый идеал алгебры $A$, в то время как $B$-инвариантность равносильна Ad-инвариантности идеала $L$ (лемма 4.2 , (iii)). Остается воспользоваться теоремой 6.3 , (ii).

6.6. СлЕДСтвиЕ. Отображение $L \mapsto \Gamma(L)$ определяет сюрғекцию множества конечномерных $Q(A, B)$-модулей $F_{1} \subset A^{*}$, содержащих $\varepsilon$, на множество биковариантных дифференциальных исчислений конечного ранга над алгеброй $A$.

ЗАмечАние. Случай $\operatorname{codim} L<\infty$ рассматривался ранее в [4]. Связь с квантовым дублем (в несколько иных контекстах) рассматривалась в [16], [5] также при $\operatorname{codim} L<\infty$. (См. [4]-[6], [15] относительно дальнейшего развития дифференциально-геометрических конщепций в алгебрах Хопфа (дифференциальные формы произвольного порядка, квантовые алгебры Ли и т.д.).)

6.7. ОпРЕДЕЛЕНИЕ. Пусть $(\Gamma, d)$ - дифференциальное исчисление первого порядка с оператором $\delta x=x_{1} \otimes x_{2}$ (см. п. 6.1.), где $x \in$ Г. Положим

$$
f^{\prime} x=f\left(x_{1}\right) x_{2}
$$

для элементов $f \in A^{*}$.

Заметим, что отображение $f \mapsto f^{\prime}$ определяет антипредставление для каждой дуальной биалгебры $B \subset A^{*}$. Действительно, для каждой пары элементов $a, b \in B$ имеем

$$
b^{\prime} a^{\prime} x=a\left(x_{1}\right) b^{\prime} x_{2}=a\left(x_{1}\right) b\left(x_{2}\right) x_{3}=(a b)\left(x_{1}\right) x_{2}=(a b)^{\prime} x,
$$

где использовано правило коассоциативности (6.3) для оператора $\delta^{2}=(\Delta \otimes 1) \delta=$ $(1 \otimes \delta) \delta$. Соответственно отображение (6.8) можно рассматривать как представление "противоположной" алгебры $B^{\prime}$.

6.8. ПРЕДЛОЖЕНИЕ. Пространство Г есть левый $D(A)$-модуль с операторами $a \in A, \quad \partial=b^{\prime} \in B^{\prime}$ (cм. (6.8)). Onератор $d: A \rightarrow \Gamma$ cплетает представления алгебры $B^{\prime}$ в пространствах $A, \Gamma$.

Действительно, для каждой пары $a \in A, b \in B$ имеем

$$
b^{\prime} a x=b\left(a_{1} x_{1}\right) a_{2} x_{2}=b_{1}\left(a_{1}\right) b_{2}\left(x_{1}\right) a_{2} x_{2}=b_{1}^{\prime}(a) b_{2}^{\prime}(x),
$$

т.е. операторы $\partial=b^{\prime} \in B^{\prime}$ удовлетворяют тождеству Лейбница (3.10). 
Отсюда следует, что операторы $a \in A, \partial=b^{\prime} \in B^{\prime}$ в пространстве $\Gamma$ удовлетворяют структурным соотношениям алгебры $D(A) \approx W(A)$ (теорема п. 3.11), т.е. образуют представление алгебры $D(A)$.

Ясно также, что $\delta(d x)=x_{1} \otimes d x_{2}$ для элементов $x \in A$ (см. п. 6.1). Поэтому

$$
f^{\prime}(d x)=f\left(x_{1}\right) d x_{2}=d\left(f^{\prime} x\right)
$$

для элементов $f \in A^{*}$, где $f^{\prime} \in \operatorname{End} \Gamma$ (соответственно $f^{\prime} \in \operatorname{End} A$ ) в левой (правой) части этого равенства.

ЗАмечание. Связь между алгеброй $D(A)$ и ковариантньми $A$-модулями (в частности, $(\Gamma, d))$ предполагается более детально рассмотреть в отдельной статье.

\section{§ 7. Элементы топологии}

7.1. Отметим связь дифференциальных операторов типа $(A, B)$ со слабой топологией $\sigma(A, B)$ в пространстве $A$, определяемой посредством дуальной пары $A, B$.

Пусть $\operatorname{End}_{\sigma} A$ - алгебра всех слабо непрерывных ( $\sigma(A, B)$-непрерывных) операторов в пространстве $A$. Как известно [1], оператор $a \in \operatorname{End} A$ слабо непрерывен тогда и только тогда, когда он обладает сопряженным $a^{\prime} \in \operatorname{End} B$ (см. п. 1.3). Отсюда следует, что все дифференциальные операторы типа $(A, B)$ слабо непрерывны, т.е. алгебры (1.14), (1.15) содержатся в $\operatorname{End}_{\sigma} A$.

Мы будем рассматривать также операторы $e \in \operatorname{End} A$, удовлетворяюшие соотношениям Лейбница следуюшего вида:

$$
e(x y)=\sum_{i} f_{1}^{i} x \otimes f_{2}^{i} y \equiv f_{1} x \otimes f_{2} y
$$

для всех $x, y \in A$, где символы $f_{1}, f_{2}$ отождествляются с соответствуюшими семействами операторов $f_{1}^{i}, f_{2}^{i}$ (по аналогии с п. 1.1).

7.2. ПРЕДЛОЖЕНИЕ. Для эндоморфизма $е \in$ End $A$ следующие условия равносильнь:

$(\alpha)$ оператор е слабо непрерывен $\left(e \in \operatorname{End}_{\sigma} A\right)$;

$(\beta)$ оператор е удовлетворяет $(7.1)$, где $f_{1}, f_{2} \subset \operatorname{End}_{\sigma} A$;

$(\gamma)$ оператор е удовлетворяет (7.1), где либо $f_{1} \subset \operatorname{End}_{\sigma} A$, либо $f_{2} \subset$ $\operatorname{End}_{\sigma} A$.

Действительно, если $(\alpha)$ выполняется, то мы для каждой тройки $x, y \in A, z \in$ $B$ имеем

$$
\langle e(x y), z\rangle=\langle x y, w\rangle=\left\langle x, w_{1}\right\rangle\left\langle y, w_{2}\right\rangle,
$$

где $w=e^{\prime} z=w_{1} \otimes w_{2}$. Условимся считать (см. п. 1.5), что элементы $w_{2}^{i} \quad(i=$ $1, \ldots, n)$ суть базисные элементы пространства $B$, и пусть $y_{i}(i=1, \ldots, n)-$ дуальная система в $A$, т.е. $\left\langle y_{j}, w_{2}^{i}\right\rangle=\delta_{i j}$ для всех $i, j=1, \ldots, n$. Тогда имеем

$$
\left\langle x, w_{1}^{i}\right\rangle=\left\langle e\left(x y_{i}\right), z\right\rangle
$$


для всех $i=1, \ldots, n$. Соотношение (7.2) принимает следующий вид:

$$
e(x y)=\sum_{i=1}^{n} a_{i}(x) b_{i}(y)
$$

где операторы $a_{i}(x)=e\left(x y_{i}\right), b_{i}(y)=\left\langle y, w_{2}^{i}\right\rangle \cdot 1$ слабо непрерьвны.

Таким образом, импликация $(\alpha) \Rightarrow(\beta)$ доказана. Импликация $(\beta) \Rightarrow(\gamma)$ тривиальна. Импликация $(\gamma) \Rightarrow(\alpha)$ вытекает из равенства

$$
e(x)=f_{1}(x) f_{2}(1)=f_{1}(1) f_{2}(x)
$$

для операторов (7.1).

7.3. СлЕДСтвИЕ. Эндоморфизмы $e \in \operatorname{End}_{\sigma} A$ удовлетворяют соотношениям следующего вида:

$$
\Delta[e(x)]=e_{1}(x) \otimes e_{2}(x)
$$

əдe $e_{1}, e_{2} \subset \operatorname{End}_{\sigma} A$.

Действительно, (7.6) сопряжено соотношению (7.1) в алгебре $B$.

7.4. СлЕДСтвиЕ. Алгебра $E=\operatorname{End}_{\sigma} A$ есть коалгебра относительно каждого из следующих отображений:

$$
\Delta e=e_{1} \otimes e_{2}, \quad \Delta^{\prime} e=f_{1} \otimes f_{2}
$$

в обозначениях (7.1), (7.6). При этом операторы $\Delta, \Delta^{\prime}$ связаны соотношением сопряденности следующего вида:

$$
\Delta^{\prime} e=\left(\Delta e^{\prime}\right)^{\prime}
$$

для всех $е \in E$. Здесь $e \mapsto e^{\prime}-$ операция сопряжения, $\operatorname{End}_{\sigma} A \rightarrow \operatorname{End}_{\sigma} B$.

7.5. ПрЕДЛОЖенИЕ. Преобразование $\Delta$ совпадает с одноименным оператором (1.3) на әлементах $A \cup \bar{A}$ в алгебре $Е$. Преобразование $\Delta$ действует на векторные поля $\partial(x)=f * x, \bar{\partial}(x)=x * f$ следующим образом:

$$
\Delta \partial=1 \otimes \partial, \quad \Delta \bar{\partial}=\bar{\partial} \otimes 1 .
$$

Преобразование $\Delta^{\prime}$ действует на векторные поля $y=\partial, \bar{\partial}$ по правилу

$$
\Delta^{\prime} y=y_{1} \otimes y_{2}
$$

в обозначениях (1.12).

Доказательство сводится к элементарной проверке. Например, имеем

$$
\Delta \partial(x)=\Delta\left(x_{1} f\left(x_{2}\right)\right)=x_{1} \otimes x_{2} f\left(x_{3}\right)=(1 \otimes \partial) \Delta x
$$

для всех $x \in A$. 
ЗАмечание. Ясно также, что $\Delta^{\prime} a=a \otimes 1, \Delta^{\prime} \bar{a}=1 \otimes \bar{a}$ для всех $a \in A$.

7.6. ПрЕДЛОЖенИЕ. Каждая из алгебр $D(A), \bar{D}(A), D(\bar{A}), \bar{D}(\bar{A})$ совпадает с множеством всех $e \in E$, удовлетворяющих соответственно соотношению $\Delta^{\prime} e \in E \otimes B^{\prime}, \quad \Delta^{\prime} e \in E \otimes \bar{B}^{\prime}, \Delta^{\prime} e \in B^{\prime} \otimes E, \Delta^{\prime} e \in \bar{B}^{\prime} \otimes E$.

Действительно, все эти соотношения необходимы. С другой стороны, пусть $\Delta^{\prime} e \in E \otimes B^{\prime}$. Отсюда согласно (7.5) имеем $e \in A B^{\prime}=D(A)$. Остальные соотношения рассматриваются аналогично.

7.7. Алгебра $A^{*}$. Пусть $A$ - алгебра Хопфа. Заметим, что операция сопряжения позволяет наделить $A^{*}$ структурой топологической алгебры Хопфа с обычным умножением и коумножением $\Delta: A^{*} \rightarrow A^{*} \widehat{\otimes} A^{*}$, где символ $F \widehat{\otimes} G$ означает локально конечное расширение подмножества $F \otimes G$ (естественно вложенного в $\left.(A \otimes A)^{*}\right)$, т.е. множество локально конечных функционалов следуюшего вида:

$$
\varphi=\sum_{i} f_{i} \otimes g_{i}
$$

с элементами $f_{i} \in F, g_{i} \in G$. При этом $\Delta$ есть гомоморфизм алгебры $A^{*}$ в алгебру $A^{*} \widehat{\otimes} A^{*}$, умножение в которой определяется как покомпонентное умножение рядов (7.11). Более того, элементы $\Delta f\left(f \in A^{*}\right)$ допускают запись (7.11) с локально конечньгм семейством $f_{i}$ (аналогично $g_{i}$ ).

7.8. Алгебра $D^{\infty}(A)$. Сохраним обозначения п. 7.7, и пусть $D^{\infty}(A)$ - векторное пространство линейных операторов следующего вида:

$$
e=\sum_{i} a_{i} \partial_{i}
$$

где $a_{i} \in A$ и семейство $\partial_{i}=b_{i}^{\prime}\left(b_{i} \in A^{*}\right)$ локально конечно. Используя конструкцию п. 7.7, легко установить, что $D^{\infty}(A)$ есть алгебра (подалгебра в End $A$ ).

Операторы (7.12) можно рассматривать как дифференциальные операторы "бесконечного порядка" в End $A$. Для алгебры $D^{\infty}(A)$ имеют место естественные аналоги теорем пा. $2.2,3.1$. Конструкция $\S 6$ также естественно переносится на алгебpy $D^{\infty}(A)$.

\section{Список литературы}

1. Бурбаки Н. Топологические векторные пространства. М.: ИЛ, 1959.

2. Диксмье ЖК. Универсальные обертывающие алгебры. М.: Мир, 1977.

3. Желобенко Д. П. Дифференциальные операторы в градуированных алгебрах // Изв. РАН. Сер. матем. 1996. Т. 60. № 2. С. 49-72.

4. Ashieri P., Schupp P. Vector fields on quantum groups. Preprint q-alg./9505023. Univ. di Pisa, 1995.

5. Bernard D. Quantum Lie algebras and differential calculus on quantum groups // Progr. Theor. Phys. Suppl. 1990. V. 102. P. 49-66.

6. Bonechi F., Giachetti R., Maciocco R., Sorace E., Tarlini M. Quantum double and differential calculi. Preprint q-alg./9507004. Univ. di Firenze, 1995. 
7. Chari V., Pressley A. A Guide to Quantum Groups. Cambridge U. P., 1995.

8. Drinfeld V.G. Quantum Groups // Proc. Intern. Congress of Math. Berkeley, 1986. Providence: AMS, 1987. (Рус. перевод: Зап. научных семин. ЛОМИ. 1986. Т. 155. C. 18-49.)

9. De Concini C., Lybashenko V. Quantum function algebra at roofs of $1 / /$ Adv. in Math. 1994. V. 108. № 2. P. 205-262.

10. Joseph A. Quantum Groups and Their Primitive Ideals. N. Y.: Springer, 1995.

11. Kashiwara $M$. Crystalizing the $q$-analogue of universal enveloping algebras // Comm. Math. Phys. 1990. V. 122. P. 249-260.

12. Kashiwara M. Global crystal bases of quantum groups. Preprint RIMS. Kyoto Univ., 1992.

13. Lusztig G. An Introduction to Quantum Groups. Birkhäuser, 1995.

14. Woronowicz S.L. Tannaka-Krein duality for compact matrix pseudogroups. Twisted $S U(N)$ groups // Invent. Math. 1988. V. 93. P. 35-76.

15. Woronowicz S. L. Differential calculus on compact matrix pseudogroups (quantum groups) // Comm. Math. Phys. 1989. V. 122. P. 125-170.

16. Woronowicz S. L. Quantum deformation of Lorentz group // Comm. Math. Phys. 1990. V. 130. P. 381-431.

17. Zhelobenko D.P. Crystal bases and the problem of reduction in classical and quantum modules // Adv. in Math. Sci. AMS Transl. (2). 1995. V. 169. P. 183-202.

18. Zhelobenko D. P. Differential operators on quantum groups and graded algebras // Proc. XXI Intern. Coloquium Group Theor. Methods in Physics. Goslar, 1996.

19. Новиков С. П. Различные удвоения алгебр Хопфа. Алгебры операторов на квантовых группах. Комплексные кобордизмы // УМН. 1992. Т. 47. № 5. С. 189-190.

20. Zhelobenko D. P. New aspects of differential calculus on quantum groups // Proc. V Wigner Symposium. Wien, 1997. 